\title{
XML-based EDI Document Processing System with Binary Format Mapping Rules
}

\author{
Chang-Su Kim ${ }^{1}$ and Hoe-Kyung Jung ${ }^{2 *}$, Member, KIICE \\ ${ }^{1}$ Industry-Academic Cooperation Foundation, Paichai University, Daejeon 302-735, Korea \\ ${ }^{2}$ Department of Computer Engineering, Paichai University, Daejeon 302-735, Korea
}

\begin{abstract}
Recently, the magnitude of electronic data interchange (EDI) document processing for the handling of port logistics is abruptly being increased. The existing system processes EDI documents in a script mode, but due to a complicated script preparation procedure and low document processing efficiency, it cannot meet the demand as the usage flow of documents increases. In this paper, an EDI electronic document processing system was designed and implemented in a document scanner and mapper, which are binary form electronic document processing tools and do not require script files during the conversion of extensible markup language (XML)-based electronic documents. This new system has the merits of XML features during reading and writing with improved speed, usage convenience, and good portability on systems when compared to the conventional ones.
\end{abstract}

Index Terms: XML, EDI, B2B, Document processing system

\section{INTRODUCTION}

Currently, varieties of electronic data interchange (EDI) electronic document processing systems have been set up and are being used. In the existing EDI document processing system, an interpreter mode using a script is used to convert the document, resulting in a long processing time and operational inefficiency. It also uses a closed network using a value added network (VAN) that is within a limited service range from the service provider, rendering the system compatibility inferior.

Recently, because EDI document processing systems using web protocols are in demand, and various types of mapping are necessary during extensible markup language (XML)-based EDI document processing as well as numerous other protocols, document processing types, a graphics tool is required during the mapping process [1-3].
In this paper, in order to decrease the conversion time and increase the operation efficiency of the existing EDI documents, we designed and implemented an EDI document processing system that can convert binary type XML-based EDI documents on which a mapping tool is applied to make automatic rule generation during web-based B2B gateway management into a user-defined flat file and then the flat file into an XML-based EDI electronic document.

\section{RELATED RESEARCH}

\section{A. Electronic Data Interchange (EDI)}

EDI is a structured standard of documents that are exchanged among computers of enterprises or of public organizations following a mutually-agreed data format and

Received 15 June 2012, Revised 23 July 2012, Accepted 30 July 2012

*Corresponding Author E-mail: hkjung@pcu.ac.kr 
communication standard via standardized uniform formats and a uniform code system. Though EDI standards vary depending on the localities and purposes, the United Nations rules for Electronic Data Interchange for Administration, Commerce and Transport (UN/EDIFACT) enacted by the UN has been well established as a standard grammar and recognized internationally [1].

\section{B. Existing EDI Document Processing System}

In the existing EDI document processing mode [4], by using a scan script file to scan the document, the document processor maps the EDI document information as a flat file as the user-defined rule using a user-defined flat file mapping script file.

With this method, since documents have to go through the process to read and use the script as well as EDI documents or flat files, the system cannot reduce the document reading load. Furthermore, by reading the document, this information is stacked inside memory. In case any mishap occurs during document processing, information stacked inside memory would instantly get lost. Consequently, the integrity of document cannot be guaranteed even when the mishap is sorted out.

\section{Analysis of Existing EDI System}

\section{1) Method of Processing XML-based EDI Electronic Documents}

Existing research on EDI electronic document processing has been mainly on conversion between EDI standard documents and the flat file type, and the research based on $\mathrm{XML}$ is scarce. Also, in document processing, EDI electronic document information is loaded in the memory using a scan script file for electronic document scanning, and then this loaded information on the memory is mapped as a flat file according to the rules that were defined by users using the user-defined flat file mapping script file. This method includes a process of script reading and use, and another process of reading either EDI electronic documents or flat files. Ultimately, the load for document reading could not be reduced [5].

\section{2) B2B Gateway Control System}

The existing B2B gateway control system is being used as an application program base. This process, therefore, impairs versatility, causing a portability problem. It is also true that many users want to control EDI on web protocols owing to the recent development of the internet.

\section{3) Mapping Tool for Rule Generation}

For the conversion among many EDI electronic documents, a mapping rule must be created. Until now, it has been done manually by the administrator. The reality is that tremendous time is consumed because of the long time for the mapping, causing large errors.

\section{Requirements}

As can be seen from the review above, it is clear that an XML-based system is required in EDI electronic document processing. Also, binary type processing, not the existing processing method using a script, is needed. The system needs to be developed in such a way that the B2B gateway system control is transferred to a web-based one and that the mapping tool can automatically create a mapping rule graphically replacing the conventional one that is manually operated. Therefore, an XML-based EDI system that accommodates these requirements was designed and implemented in this study.

\section{DESIGNING THE EDI DOCUMENT PROCESSING SYSTEM}

In the XML-based EDI electronic document processing system, trading partners, who are the users of XML EDI electronic documents, convert these in the electronic document processing system proposed in this study and then send them again to end users. When XML EDI electronic documents are received by the user, the processing system extracts recorded sent and received information in electronic documents and converts them to user-defined flat files.

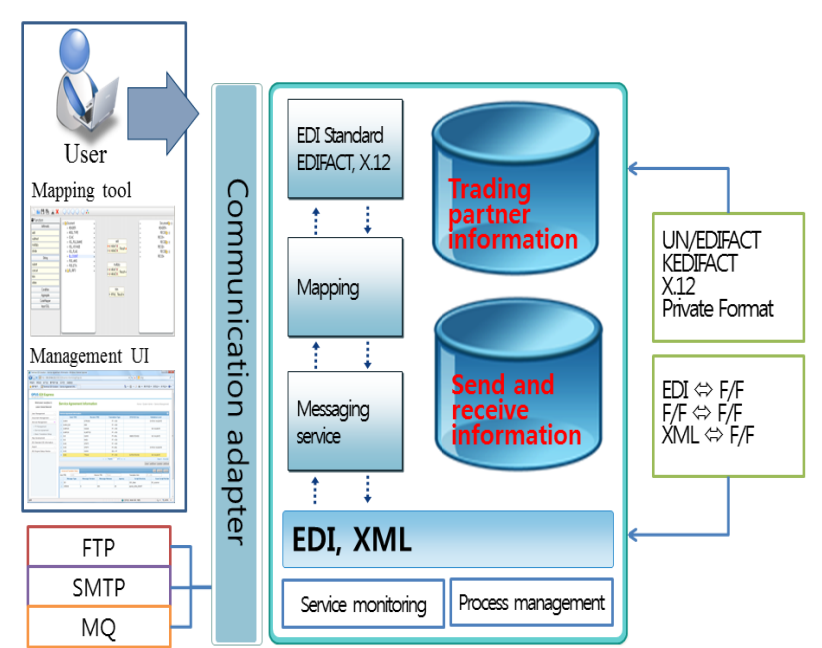

Fig. 1. Structural diagram of electronic data interchange (EDI) document processing system based on extensible markup language (XML). FTP: file transfer protocol, SMTP: simple mail transfer protocol, MQ: message queuing, F/F: flat file, UN/EDIFACT: United Nations rules for Electronic Data Interchange for Administration, UI: user interface. 
Fig. 1 shows a structural diagram of the EDI document processing system based on XML designed in this paper.

The information referred to during this time is information on a trading partner and EDI standard information. This information becomes converted from XML to a flat file or from a flat file to XML electronic documents through the management process to create a mapper on which mapping-related rules are designated.

The converted electronic document is transmitted through the internet, and file transfer protocol (FTP), simple mail transfer protocol (SMTP), and message queuing (MQ) are supported for electronic document sending and receiving to/from trading partners. Also, there is a mapping tool in which rules for various document conversions can be automatically generated. Additionally, it is equipped with a web-based management system to control this tool over the web.

\section{A. Module Construction for XML-based EDI Document Processing System}

In the XML-based EDI electronic document conversion system, there are servers to convert XML EDI electronic documents, document sending and receiving modules, an engine that converts EDI electronic documents, and a client module for operation management. Fig. 2 shows the components of this system.

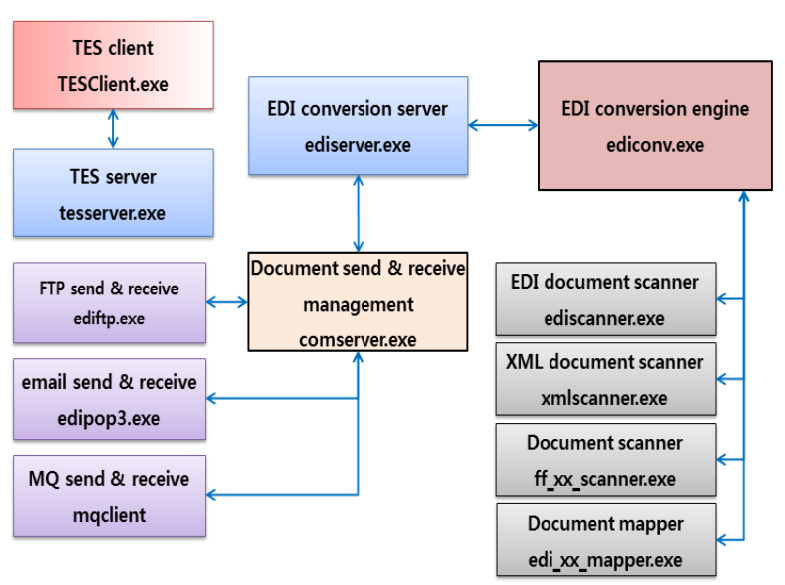

Fig. 2. e-document processing system modules. EDI: electronic data interchange, XML: extensible markup language, FTP: file transfer protocol, MQ: message queuing, TES: transportation execution systems.

\section{B. Designing the XML-to-Flat File Conversion Program}

The conversion process of XML to flat files is shown in Fig. 3. In this process, XML documents are inputted, scanned, and conversion is executed. During this time, various conditions for the conversion are being referred to through transaction processing (TP) information for document conversion and service agreement information, etc. A mapper in which mapping information is already loaded is also referred to in order to convert to a flat file. Mapping is done as a user-defined type and then saved.

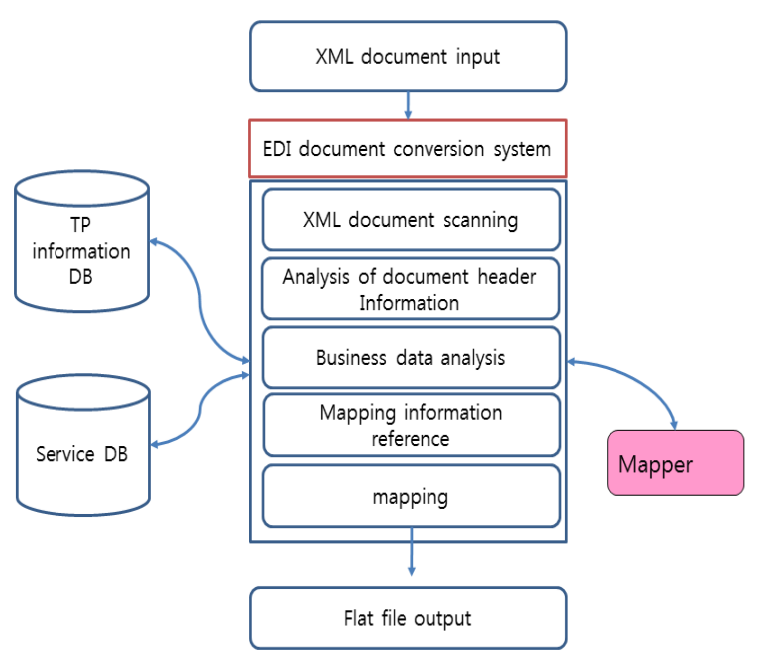

Fig. 3. Extensible markup language $(X M L)$-to flat file conversion process EDI: electronic data interchange, TP: transaction processing.

\section{Designing the Flat File-to-XML Conversion Program}

The process of flat file-to-XML conversion is shown in Fig. 4. The received flat file that needs to be converted is scanned, the required information is obtained, and the scan and conversion method are extracted by referring to the service agreement information, and finally business data are extracted by calling the scanner. The extracted business data are applied on the mapper and stored as an XML/BIZ file in case mapper tries converting the file into a business file instead of an XML file. The extracted business data are converted into an XML file through the optimization part of the engine.

\section{Mapping Tool}

In an EDI document information processing system, many conversions take place among various EDI electronic documents, so a large amount of time and many complicated steps are required to define rules manually for the conversion. Therefore, a mapping rule which can run to generate rules graphically between the converted documents was designed and implemented in this study. 


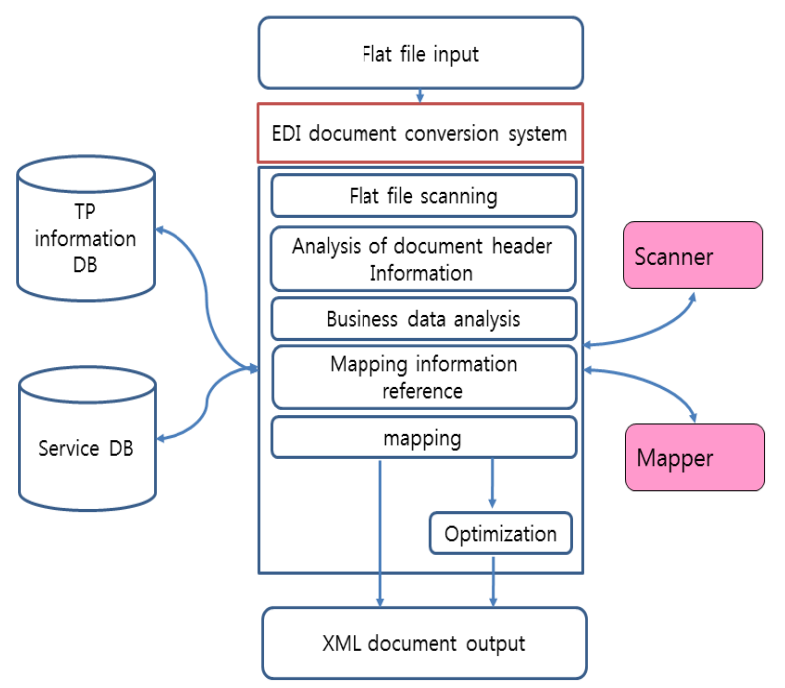

Fig. 4. Flat file-to-extensible markup language $(\mathrm{XML})$ conversion process. EDI: electronic data interchange, TP: transaction processing.

\section{IMPLEMENTATION OF XML BASED EDI DOCUMENT PROCESSING SYSTEM}

The system was developed in the $\mathrm{C}$ language and Visual $\mathrm{C}++$, and the system core part for XML-based EDI electronic document processing was specifically configured to enable cross compiling to be used in Windows and Linux. The database for system operation was designed to have a flexible system operation using a relational database.

The XML receiving window, inputted XML document, and conversion window with XML business data for converting the inputted XML document by XMLScanner are shown in Figs. 5-7, respectively.

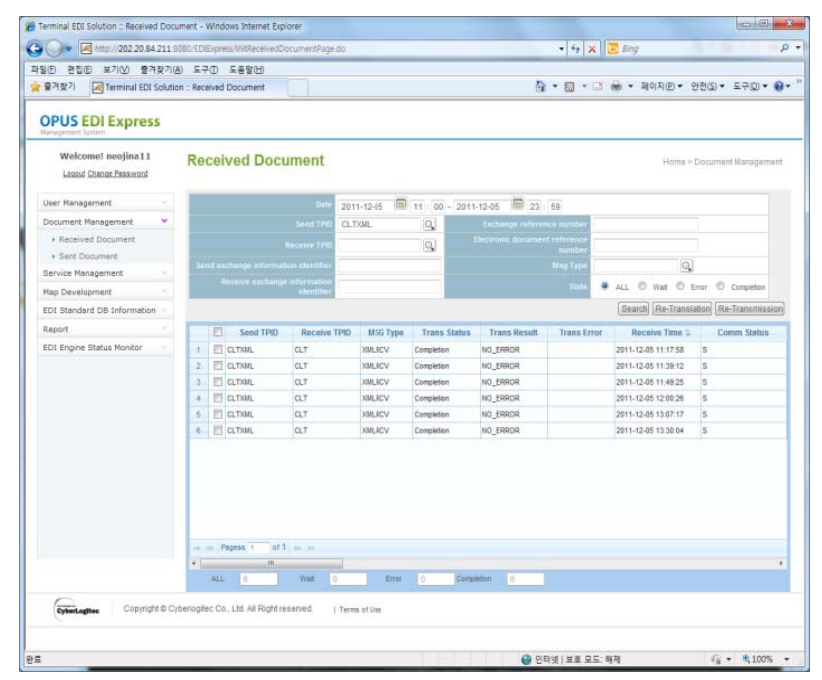

Fig. 5. Screen of extensible markup language $(X M L)$ receive processing.

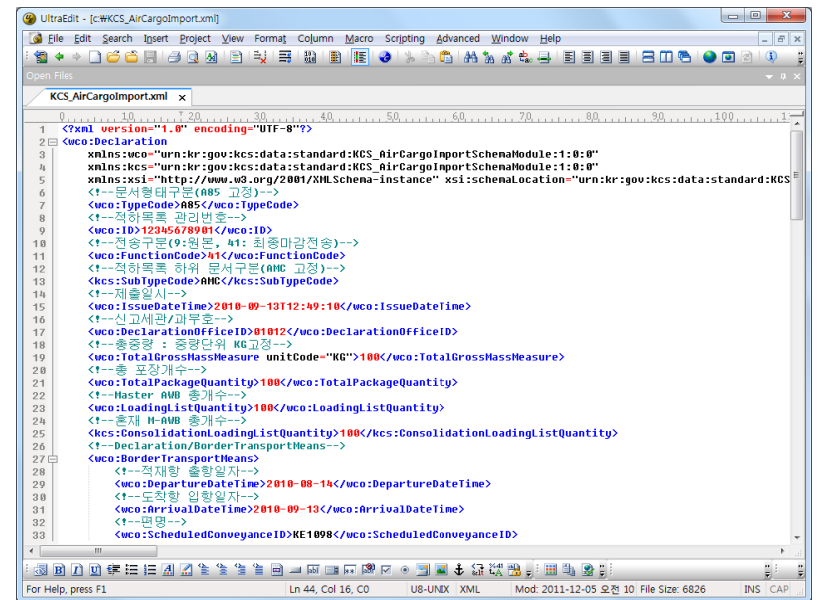

Fig. 6. Inputted extensible markup language (XML) document.

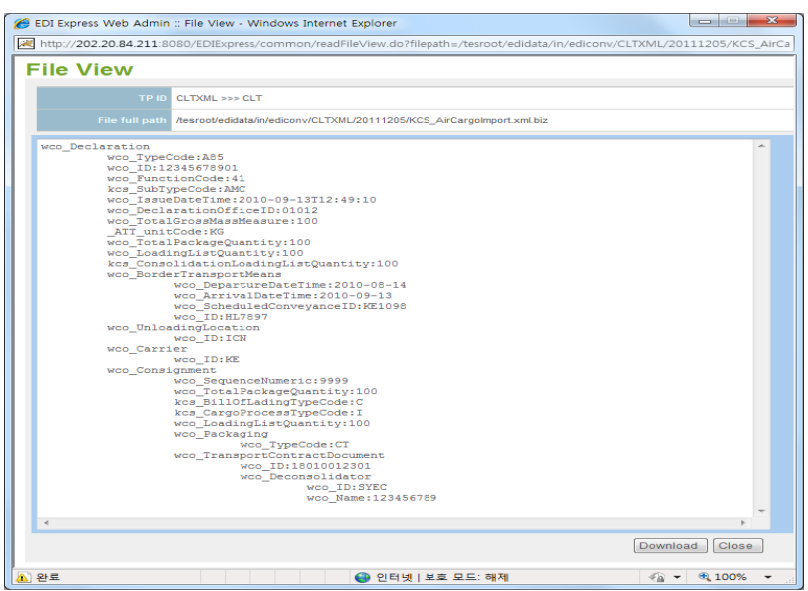

Fig. 7. Extensible markup language $(X M L)$ business data.

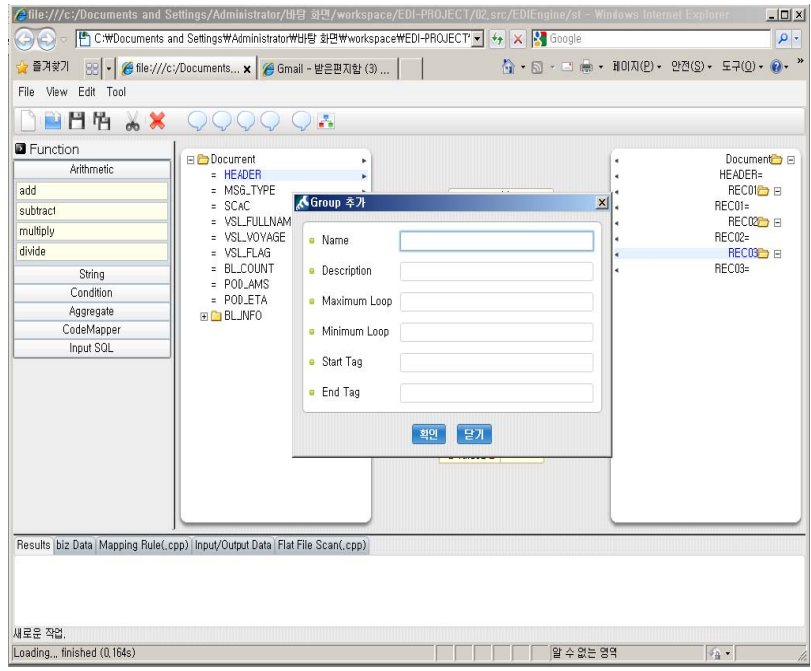

Fig. 8. Screen of mapping tool. 


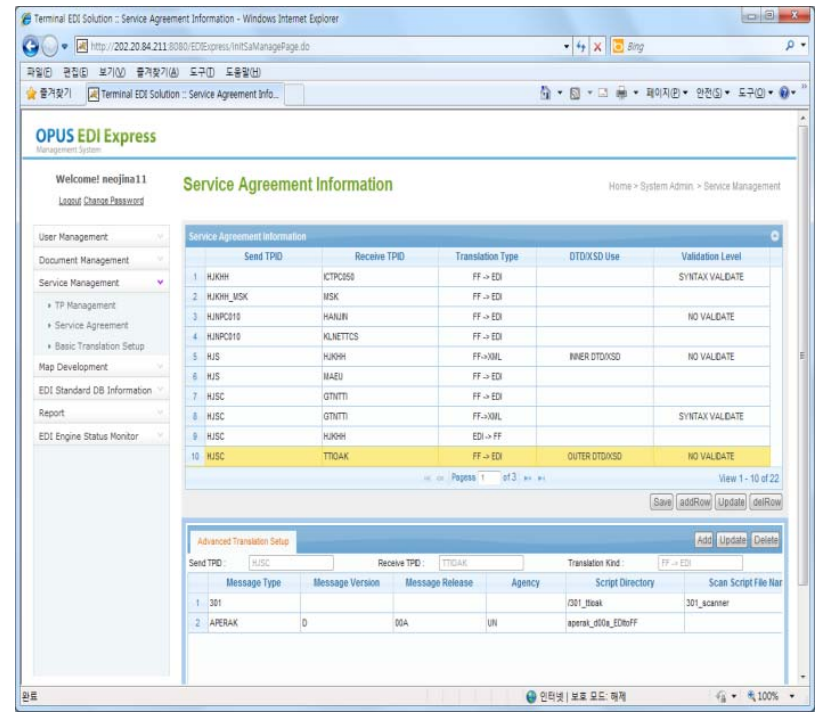

Fig. 9. Screen of service agreement information.

The window of the web-based control system can be controlled since a variety of information, including agreement, is based on the web, as shown in Fig. 8. Input and modification are possible and judged as easier than the existing application program since it can be accessed from anywhere and anytime as long as the internet is available. Also, because rules are automatically generated by defining the rules graphically as shown in Fig. 9, rule generation between documents is easy.

\section{CONCLUSIONS}

In this study, an XML-based EDI electronic document system was designed and implemented in such a way that in the process mode, document scanning and mapping with a binary type without using an existing script mode was proposed. Also, a web-based EDI control system was designed in order to allow accessibility from anywhere at any time. By providing graphical conversion tools to create conversion rules between varieties of EDI documents, rules can easily be created, and by inspecting the rules automatically, validation of the rules is also possible.

Since the XML-based EDI electronic document processing system proposed in this paper inter-converts between Linux and Windows, it would be more useful than existing EDI electronics document processing systems by making transplant possible even among various systems.

Also, it could be utilized as a B2B gateway system with all the merits of XML characteristics such as faster speed and better usage convenience compared to the existing EDI electronic document processing system as well as portability of the system.

As a future research task, research about an expandable system and environment that can make a scanner and mapper using a graphical user interface needs to be carried out.

\section{REFERENCES}

[1] J. Ham, “A study on EDI e-document processing system for port logistics,” Journal of Information and Communication Engineering, vol. 15, no. 5, pp. 1081-1086, 2011.

[2] F. Attarchi and N. Fallah, "Design procedure for an EDI System," Ultrapure Water Journal, vol. 21, no. 5, pp. 29-34, 2004.

[3] G. Yixun, “EDI system based on client/server architecture,” Journal of Xiamen University, vol. 37, no. 5, pp. 662-667, 1998.

[4] P. Verstege, J. Brun, C. Cussac, J. M. Dangreaux, C. Heilbuth, H. Mehnen, A. Ritz, L. Vaessen, and C. Williams, "An audit framework for EDI-related systems," Proceedings of the 9th International Conference on EDI-IOS, Bled, Slovenia, pp. 556-570, 1996.

[5] T. Xu, Y. Jin, and L. Li, "Designing and implementation of the XML-based symmetrical web-EDI system,” Computer Integrated Manufacturing Systems, vol. 7, no. 10, pp. 53-56, 2001.

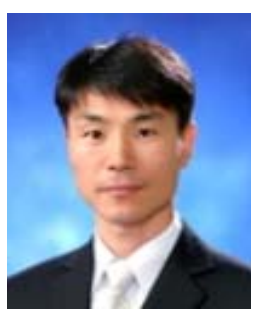

Chang Su Kim

received B.S., M.S., and Ph.D. degrees from the Department of Computer Engineering of Paichai University, Korea, in 1996, 1998, and 2002, respectively. From 2005 to 2012, he worked for the Department of Internet at Chungwoon University as a professor and he has worked in the Business Support Division at the Daejeon Technopark. Since 2012, he has worked at the Industry-Academic Cooperation Foundation at Paichai University, where he works as an industry-academic cooperation professor. His current research interests include multimedia document architecture modeling, web 2.0, and the semantic web. 


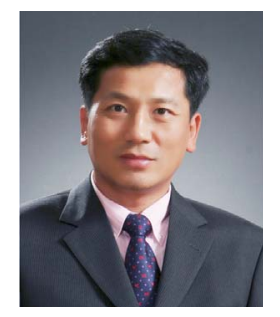

Hoe Kyung Jung

received the B.S degree in 1987 and Ph. D. degree in 1993 from the Department of Computer Engineering of Kwangwoon University, Korea. From 1994 to 2005, he worked for ETRI as a researcher. Since 1997, he has worked in the Department of Computer Engineering at Paichai University, where he now works as a professor. His current research interests include multimedia document architecture modeling, information processing, information retrieval, and databases. 